\title{
Aphasia Rapid Test: Estudos de Tradução, Adaptação e Validação para a População Portuguesa
}

\section{Aphasia Rapid Test: Translation, Adaptation and Validation Studies for the Portuguese Population}

\author{
Miguel TÁBUAS-PEREIRA ${ }^{1}{ }^{1}$, Sandra FREITAS ${ }^{2}$, José BEATO-COELHO ${ }^{1}$, Joana RIBEIRO ${ }^{1}$, Joana PARRA ${ }^{1}$, \\ Cristina MARTINS ${ }^{3}$, Miguel SILVA ${ }^{4}$, Maria Assunção MATOS ${ }^{5,6}$, Ana Rita NOGUEIRA ${ }^{7}$, Fernando SILVA ${ }^{1}$, \\ João SARGENTO-FREITAS ${ }^{1}$, Gustavo CORDEIRO ${ }^{1}$, Luís CUNHA ${ }^{1,4}$, Isabel SANTANA ${ }^{1,4}$
}

Acta Med Port 2018 May;31(5):265-271 • https://doi.org/10.20344/amp.9090

\section{RESUMO}

Introdução: As baterias clássicas de caracterização de afasia são demasiado longas para serem utilizadas no contexto do acidente vascular cerebral agudo ou como ferramenta de monitorização. O Aphasia Rapid Test é uma escala de 26 pontos desenvolvida como teste de cabeceira para avaliar a gravidade da afasia num doente com acidente vascular cerebral em menos de três minutos. $O$ objetivo do estudo é adaptar e validar a escala para o português europeu.

Material e Métodos: Foram avaliados 56 doentes com acidente vascular cerebral no primeiro e sétimo dia pós-acidente vascular cerebral. Ao sétimo dia, foram avaliados por dois avaliadores independentes para avaliar o acordo interavaliadores. Para estudar a validade concorrente, a 20 doentes foi aplicada também a Bateria de Avaliação de Afasias de Lisboa. A capacidade preditiva do Aphasia Rapid Test foi avaliada aos seis meses, através do valor da subescala de afasia do National Institutes of Health Stroke Scale. Resultados: A tradução para o português europeu baseou-se nas versões francesa e inglesa, respeitando a frequência de utilização das palavras. $\mathrm{O} \alpha$ de Cronbach foi de 0,796 . O coeficiente de concordância entre examinadores foi excelente $(0,985)$. A correlação entre o Aphasia Rapid Test e a Bateria de Avaliação de Afasias de Lisboa é forte $(r=-0,958, p<0,001)$. Os gráficos de BlandAltman corroboram as boas concordâncias interavaliadores e validade concorrente. O Aphasia Rapid Test no primeiro dia é preditor independente do resultado a longo prazo.

Discussão: Este estudo apresenta resultados confiáveis para o português europeu, com valores de consistência interna, concordância interavaliadores e validade concorrente adequados.

Conclusão: O Aphasia Rapid Test é um bom instrumento para avaliação e monitorização da afasia em doentes com acidente vascular cerebral.

Palavras-chave: Acidente Vascular Cerebral; Afasia/diagnóstico; Portugal; Resultado do Tratamento; Testes Neuropsicológicos

\section{ABSTRACT}

Introduction: Classical aphasia evaluation scales are too long to use in the context of acute stroke or as a monitoring tool. The Aphasia Rapid Test is a 26-point scale developed as a bedside assessment to rate aphasia severity in acute stroke patients in less than 3 minutes. We aimed to adapt and validate this scale for European Portuguese.

Material and Methods: We evaluated 56 acute stroke patients in the first and in the seventh days post-stroke. In the seventh day, patients were evaluated by two independent raters, to evaluate inter-rater agreement. To study concurrent validity, the Lisbon Aphasia Examination Battery was applied to a subset of 20 patients. The predictive ability of the Aphasia Rapid Test was assessed at six months, by the aphasia subscale of the National Institutes of Health Stroke Scale.

Results: Translation to European Portuguese was based in the French and English versions, considering the words' utilization frequency. The Chronbach's alpha was 0.796 . The concordance coefficient between the two raters was excellent (0.985). Correlation between Aphasia Rapid Test and the Lisbon Aphasia Examination Battery was strong $(r=-0.958, p<0.001)$. The study through BlandAltman graphs corroborated the good inter-rater agreement and concurrent validity of the test. The Aphasia Rapid Test score in the first day is an independent predictor of long-term outcome.

Discussion: This study provides reliable results for European Portuguese, with adequate internal consistency, inter-rater agreement and concurrent validity.

Conclusion: The Aphasia Rapid Test is a good tool for the evaluation and monitoring of aphasia in stroke patients.

Keywords: Aphasia/diagnosis; Neuropsychological Tests; Portugal; Stroke; Treatment Outcome

\section{INTRODUÇÃO}

A afasia afeta $21 \%$ a $38 \%$ dos doentes com acidente vascular cerebral (AVC). ${ }^{1}$ Tem um impacto importante na qualidade de vida do doente, ${ }^{2}$ afetando a sua atividade profissional e social, ${ }^{3}$ mas também a dos familiares e cui-

dadores. ${ }^{4}$ As baterias clássicas de caracterização de afasia de causa vascular são demasiado longas para serem utilizadas no contexto do AVC agudo 5 ou como ferramenta de monitorização. Nas situações agudas e com variação

\footnotetext{
1. Serviço de Neurologia. Centro Hospitalar e Universitário de Coimbra. Coimbra. Portugal.

2. Faculdade de Psicologia e de Ciências da Educação. Universidade de Coimbra. Coimbra. Portugal.

3. Centro de Estudos de Linguística Geral e Aplicada. Departamento de Línguas, Literaturas e Culturas. Faculdade de Letras. Universidade de Coimbra. Coimbra. Portugal.

4. Faculdade de Medicina. Universidade de Coimbra. Coimbra. Portugal.

5. Serviço de Medicina Física e Reabilitação. Centro Hospitalar e Universitário de Coimbra. Coimbra. Portugal.

6. Escola Superior de Saúde. Universidade de Aveiro. Aveiro. Portugal.

7. Serviço de Medicina Interna. Centro Hospitalar e Universitário de Coimbra. Coimbra. Portugal.

$\square$ Autor correspondente: Miguel Tábuas-Pereira. miguelatcp@gmail.com

Recebido: 17 de abril de 2017 - Aceite: 30 de abril de 2018 | Copyright @ Ordem dos Médicos 2018
} 
temporal, como o AVC, pretende-se um método de avaliação rápido, enquadrável numa primeira avaliação no serviço de urgência, e que seja sensível à evolução do défice afásico ao longo do tempo. Neste contexto, surgiram alguns testes de cabeceira de aplicação rápida, mas cuja capacidade para servir de instrumento de monitorização de evolução clínica é limitada ou não foi estudada. .-8 $^{-8}$

A gravidade dos défices neurológicos no contexto do AVC é atualmente quantificada por rotina pela National Institute of Health Stroke Scale (NIHSS). ${ }^{9}$ Esta escala de cabeceira é rápida, simples e reprodutível. Tem ainda sensibilidade para detetar pequenas alterações no estado neurológico e é um bom preditor do resultado funcional, ${ }^{10}$ mas avalia apenas superficialmente a linguagem. Recentemente, o Aphasia Rapid Test (ART) ${ }^{11}$ foi proposto como um teste de cabeceira para avaliar a gravidade da afasia, baseando-se nos parâmetros neurológicos que são normalmente avaliados em contexto de AVC. O ART foi desenvolvido em França por C. Azuar et al ${ }^{11}$ estando disponível nas versões francesa e inglesa e, de acordo com os seus autores, é uma ferramenta simples e rápida, aplicável por um profissional de saúde após um curto período de treino, e que não necessita de material de apoio específico. Além disso, permite a deteção precoce e a avaliação da gravidade da afasia ao longo do tempo de internamento, possibilitando ainda um encaminhamento mais informado para um programa de terapia da fala. ${ }^{12,13}$

O nosso propósito foi desenvolver os estudos necessários à utilização deste teste em Portugal. Nesse sentido, depois de efetuada a adaptação e validação transcultural ao português europeu, estudámos as suas características psicométricas e o seu potencial para predizer a evolução a longo prazo.

\section{MATERIAL E MÉTODOS \\ Participantes}

A população do estudo consistiu em doentes internados consecutivamente no Serviço de Neurologia do Centro Hospitalar e Universitário de Coimbra, entre 1 de julho de 2014 e 31 de dezembro de 2014, por AVC isquémico agudo. Foi obtido um consentimento oral de todos os participantes ou, na impossibilidade, do familiar mais próximo. Este estudo foi aprovado pela comissão de ética. Todos os dados foram anonimizados no momento da colheita, respeitando a sua confidencialidade. Todos os procedimentos respeitaram a Declaração de Helsínquia de 1975.

Foram selecionados indivíduos que cumpriam os seguintes critérios de inclusão:

a) língua portuguesa europeia como língua materna (excluindo-se sujeitos com uso mais habitual e corrente de outra língua por períodos superiores a 10 anos);

b) sujeitos dextros (assumindo-se um padrão habitual de dominância hemisférica);

c) diagnóstico pelo neurologista assistente de AVC com alteração da linguagem;

d) lesão vascular isquémica aguda identificada na tomografia axial computadorizada crânio-encefálica. Foram considerados os seguintes critérios de exclusão:

a) alteração do estado de vigília;

b) AVC prévio;

c) incapacidade física que inviabilizasse a execução das tarefas inerentes à aplicação da escala, nomeadamente intubação endotraqueal ou outra barreira física que impedisse a produção de sons.

\section{Métodos de recolha de dados \\ Material}

O ART ${ }^{11}$ foi criado para quantificar a gravidade da afasia em contexto agudo de AVC. É composto por seis subtestes, avaliando, em menos de três minutos, os quatro parâmetros fundamentais num estudo da linguagem: compreensão, repetição, nomeação e fluência do discurso. Assim, inicialmente é pedido ao doente que execute duas ordens simples ( 0 a 2 pontos) e uma ordem complexa ( 0 a 3 pontos), permitindo avaliar a compreensão verbal simples e complexa. De seguida, é avaliada a capacidade de repetição de três palavras ( 0 a 6 pontos) e de uma frase $(0$ a 2 pontos). Posteriormente, é pedida a nomeação de três objetos de uso comum ( 0 a 6 pontos). No último parâmetro, fluência do discurso ( 0 a 4 pontos), avalia-se a produção de palavras durante um minuto através de uma tarefa de fluência verbal semântica. Para além da avaliação dos quatro parâmetros fundamentais, é ainda avaliada a disartria (0 a 3 pontos) segundo um sistema de pontuação semelhante ao do NIHSS. ${ }^{12}$ O ART possui uma amplitude de pontuação de 0 a 26 pontos, correspondendo as pontuações mais elevadas a piores desempenhos.

\section{Procedimentos}

O protocolo de avaliação incluiu a versão portuguesa do ART e o NIHSS, ${ }^{9,10}$ ambos aplicados a todos os doentes, por esta ordem e pelo mesmo avaliador (neurologistas com treino em AVC). O tempo de administração do ART foi cronometrado, desde o início da primeira tarefa até ao termo da última tarefa, tendo sido administrados todos os itens a todos os participantes, mesmo nos doentes com defeito de compreensão grave.

Os doentes foram avaliados em diversos momentos após o AVC: no primeiro ( $\pm 1 \mathrm{dia})-\mathrm{D} 1$, no sétimo ( $\pm 1 \mathrm{dia}$ ) - D7- e ainda na consulta de follow-up (entre 6 e os 12 meses após o AVC). O estudo das capacidades psicométricas incidiu sobre a avaliação efetuada no sétimo dia, implicando que um subgrupo de doentes fosse examinado por dois examinadores independentes para avaliação do acordo interavaliadores, sendo as duas avaliações realizadas com intervalos sempre inferiores a doze horas. Para avaliação da validade convergente, num subgrupo da amostra (10 doentes em D7, 10 doentes seis meses após o AVC) foram administradas quatro tarefas da Bateria de Avaliação de Afasia de Lisboa (BAAL) ${ }^{13-15}$ que permitem calcular o quociente de afasia (fluência, nomeação, compreensão auditiva e repetição). 
A capacidade preditiva do ART em termos de prognóstico aos 6 - 12 meses foi obtida, comparando-o com a subescala da afasia da NIHSS (NIHSS ${ }_{\text {afasia }}$ ), que varia entre $0 \mathrm{e}$ 3. Utilizando os resultados obtidos com esta subescala, os doentes foram divididos em dois grupos: os que apresentavam pontuação de 0 ou 1 (correspondendo a uma evolução favorável do défice afásico) e os que apresentavam 2 ou 3 (o grupo com evolução da afasia mais desfavorável).

\section{Análise de dados}

A análise estatística foi realizada com recurso ao programa Statistical Package for the Social Sciences (SPSS, version 20.0) (IBM SPSS, Chicago, IL). A caracterização da amostra e do tempo de administração foram analisados com recurso a estatísticas descritivas. O $\alpha$ de Cronbach foi considerado como indicador da consistência interna da prova e os coeficientes de correlação de Pearson utilizados para examinar a validade de construto (correlações inter-subtestes e entre subtestes e pontuação total). O acordo interavaliadores foi analisado através do coeficiente de correlação intraclasses e do kappa quadrático ajustado (que considera a distância entre os resultados obtidos pelos dois avaliadores, e não apenas a simples concordância unívoca daqueles). O mesmo kappa foi calculado para cada subteste. Foram também computados gráficos de Bland-Altman para excluir possíveis diferenças sistemáticas entre os avaliadores e outliers. A validade convergente foi investigada com recurso a coeficientes de correlação entre as pontuações obtidas nas provas administradas e a uma regressão linear simples e respetiva análise da relação linear da pontuação nos testes. Para determinar a sensibilidade do ART a pequenas mudanças com impacto na evolução, foi comparada a variação do ART entre D1 e D7 (ART em D7 - ART em D1) entre grupos de diferente evolução funcional (NIHSS ${ }_{\text {afasia }}$ de 0-1 versus 2-3) através do teste de Mann-Whitney. A capacidade de predição do ART (em D1) de o doente apresentar um NIHSS afasia $_{2} 2$ a 6 - 12 meses, foi analisada através de uma regressão logística binomial, ajustada para a idade, escolaridade e $\mathrm{NIHSS}_{\text {motor }}$ em D1 (este valor foi determinado a partir do NIHSS excluindo-se as alíneas possivelmente perturbadas por défices de linguagem: perguntas de cumprimento de comandos (item 1b), linguagem (item 9) e disartria (item 10), e por considerar por queremos estudar a capacidade de predição do ART para além dos outros défices e não como alternativa ao NIHSS, para além de evitar considerar duas vezes algumas alterações da linguagem, o que aumenta a colinearidade e diminui o impacto do ART).

\section{RESULTADOS}

\section{Adaptação transcultural para a população portuguesa}

A autorização para a realização de estudos de adaptação e validação do ART para a população portuguesa foi concedida pelos autores da prova original em 2014. De seguida, foi executado o processo proposto por Herdman et $a l^{16}$ : tradução, retradução, análise de equivalência semântica e aplicação experimental. A tradução da escala para o português europeu foi realizada por uma linguista tradutora trilingue, experiente na adaptação de testes neuropsicológicos, baseando-se na escala original em francês e na tradução dos autores para o inglês. Foi respeitada a frequência de utilização das palavras da escala original (por consulta das bases LEXIQUE, ${ }^{17}$ SUBTLEX-us ${ }^{18}$ e da linguateca), ${ }^{19}$ nos itens de repetição e de nomeação. Foi realizada a retradução para o inglês, por maior facilidade do painel de peritos e esta ser a escala de uso mais generalizado. A análise de equivalência semântica foi realizada por um painel de três peritos (dois neurologistas e um neuropsicólogo) optando-se por objetos a nomear pertencentes ao uso comum e acessíveis em ambiente hospitalar, permitindo que a escala seja aplicada sem necessidade de material de apoio específico.

\section{Caracterização sociodemográfica e clínica}

O presente estudo inclui 56 doentes (36 mulheres e 20 homens; idade média de 78,5 \pm 10,3 anos; escolaridade média de 3,2 $\pm 2,9$ anos) internados por AVC agudo com afasia. Na primeira avaliação após o AVC, os doentes

Tabela 1 - Coeficientes de correlação entre subtestes da ART com a pontuação total

\begin{tabular}{|c|c|c|c|c|c|c|c|}
\hline & $\begin{array}{l}\text { Ordens } \\
\text { simples }\end{array}$ & $\begin{array}{c}\text { Ordens } \\
\text { complexas }\end{array}$ & $\begin{array}{c}\text { Repetição } \\
\text { de palavras }\end{array}$ & $\begin{array}{l}\text { Repetição } \\
\text { de frases }\end{array}$ & Nomeação & Disartria & Fluência \\
\hline $\begin{array}{c}\text { Ordens } \\
\text { complexas }\end{array}$ & $r=0,80^{*}$ & & & & & & \\
\hline $\begin{array}{l}\text { Repetição } \\
\text { de palavras }\end{array}$ & $r=0,80^{*}$ & $r=0,76^{*}$ & & & & & \\
\hline $\begin{array}{l}\text { Repetição } \\
\text { de frases }\end{array}$ & $r=0,76^{*}$ & $r=0,78^{*}$ & $r=0,88^{*}$ & & & & \\
\hline Nomeação & $r=0,71^{*}$ & $r=0,76^{*}$ & $r=0,91^{*}$ & $r=0,87^{*}$ & & & \\
\hline Disartria & $r=0,68^{*}$ & $r=0,67^{*}$ & $r=0,78^{*}$ & $r=0,71^{*}$ & $r=0,77^{*}$ & & \\
\hline Fluência & $r=0,60^{*}$ & $r=0,79^{*}$ & $r=0,73^{*}$ & $r=0,64^{*}$ & $r=0,81^{*}$ & $r=0,59^{*}$ & \\
\hline Total & $r=0,83^{*}$ & $r=0,87^{*}$ & $r=0,96^{*}$ & $r=0,91^{*}$ & $r=0,96^{*}$ & $r=0,83^{*}$ & $r=0,83^{*}$ \\
\hline
\end{tabular}


apresentaram uma pontuação média no ART de 13,0 $\pm 9,0$ e no NIHSS de 9,6 $\pm 8,3$.

\section{Avaliação do tempo de administração}

A média da duração de administração da escala (incluindo a tarefa de fluência com duração de 60 s) foi medida nos primeiros 25 doentes (14 mulheres e 11 homens; idade média de 71,9 \pm 14,4 anos; escolaridade média de 4,4 anos), e foi de dois minutos e 34,8 segundos (desvio padrão $=50,4 \mathrm{~s})$.

\section{Avaliação das características psicométricas}

Para a análise da consistência interna foram consideradas as pontuações totais no ART obtidas na avaliação ao sétimo dia, tendo-se observado um $\alpha$ de Cronbach de 0,796 . Foi possível verificar que este valor não aumentou com a exclusão de nenhum item da prova.

Relativamente à validade de construto foram observados coeficientes de correlação positivos e estatisticamente significativos entre as pontuações obtidas nos subtestes (entre 0,601 e 0,908) e entre as pontuações nos subtestes e a pontuação total no ART (entre 0,832 e 0,960) (Tabela 1).

$\mathrm{O}$ grau de acordo interavaliadores revelou-se excelente, com um coeficiente de concordância entre os dois examinadores de 0,985 [IC 95\% $=(0,974,0,991) ; p<0,001$, Fig. 1A]. O $k_{w}$ correspondente à escala foi de 0,712 [IC $95 \%$ $=(0,676,0,748) ; p<0,001]$ e os $k_{w}$ para cada subteste variaram entre 0,811 (compreensão de ordens complexas) e 0,956 (nomeação). O gráfico de Bland-Altman (Fig. 1B) mostra que a reprodutibilidade do ART é estável em todos os níveis de gravidade de afasia e que uma diferença de mais de 3,5 pontos indica uma diferença significativa na classificação da gravidade de afasia.

A validade concorrente foi explorada num subgrupo de 20 doentes (12 mulheres e oito homens; idade média de
$72,2 \pm 13,8$ anos; escolaridade média de $4 / 5$ anos), metade dos quais com afasia crónica (AVC há mais de seis meses). As Fig.s 2A e 2B mostram a relação das pontuações totais nas provas (ART e BAAL). A regressão linear mostrou um $R^{2}$ de $0,92, \beta_{\mathrm{a}}=-0,958, p<0,001$. A correlação entre as duas escalas foi de $r=-0,96(p<0,001)$.

Os doentes apresentavam em média ART de 14,7 ( \pm 8,7 ), variando entre e 2 e 26 . Quanto ao quociente de afasia da BAAL, a média foi de $51.8( \pm 36,8)$, variando entre 0 e 98,44 .

Em termos de dimensões avaliadas, verificaram-se também correlações significativas moderadas a muito fortes: compreensão $(r=-0,76, p<0,001)$, repetição $(r=-0,98$, $p<0,001)$, nomeação $(r=-0,86, p<0,001)$ e fluência $(r=$ $-0,62, p=0,003)$.

\section{Capacidade da escala para predizer a evolução a longo prazo}

Foi possível obter uma avaliação longitudinal completa (D1 e D7 e fase crónica) em 42 doentes (26 mulheres, 16 homens; idade média de $78,3 \pm 11,8$ anos). De referir que no primeiro momento da avaliação (D1), os valores do ART correlacionam-se estatisticamente com a pontuação no NIHSS $(r=0,86, p<0,001)$, mas não com o sexo $\left(r_{p b}=\right.$ $-0,17, p=0,281)$, idade $(r=0,19, p=0,238)$ ou escolaridade $(r=-0,09, p=0,624)$.

A comparação da variação do ART entre D1 e D7 (Fig. 3) nos dois grupos de evolução funcional (NIHSS ${ }_{\text {afasia }}=0$ 1 , NIHSS afasia $=2-3$ ) demonstrou que os grupos têm uma diferença com significado estatístico $(p=0,001)$. Passaram de uma mediana de 7,0 (IQR $=6,5)$ para 3,0 $($ IQR $=5,0)$ no grupo de melhor prognóstico $\left(\right.$ NIHSS $_{\text {afasia }} \leq 1$ ) e de 26,0 $(\mathrm{IQR}=2,0)$ para $26,0(\mathrm{IQR}=3,0)$ no grupo de pior prognóstico $(35,7 \%$ da amostra).
A

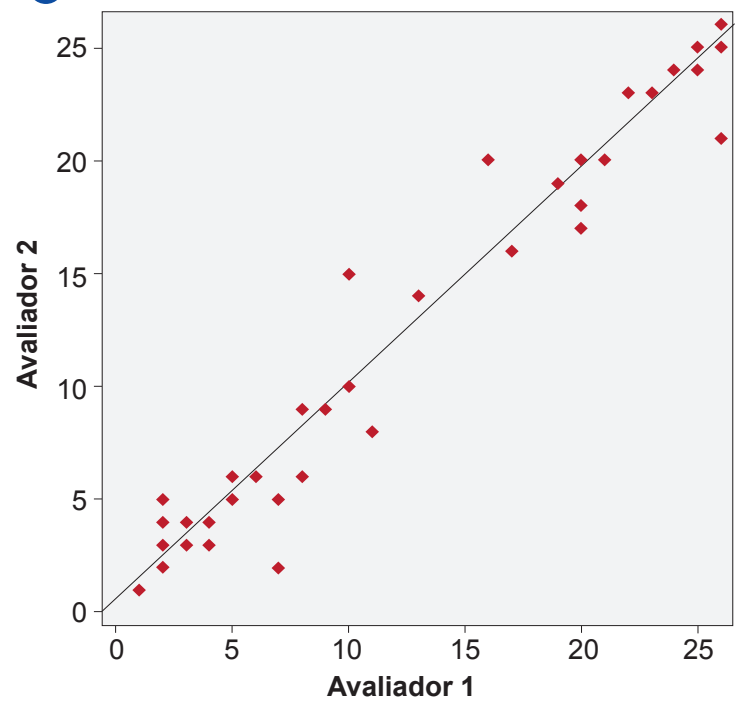

B

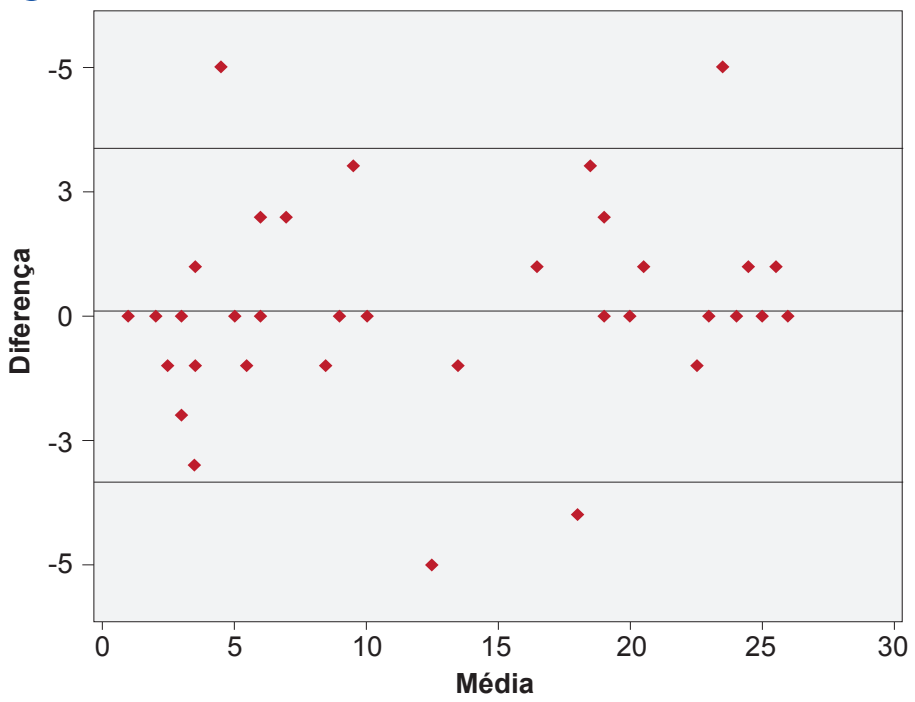

Figura 1 - (A) Distribuição dos valores do ART para cada doente segundo o avaliador 1 e o avaliador 2, o que demonstra elevada concordância interobservadores do ART demonstrada pelo coeficiente de concordância de 0,985; (B) Respetivo gráfico de Bland-Altman representando a concordância interobservadores do ART. 


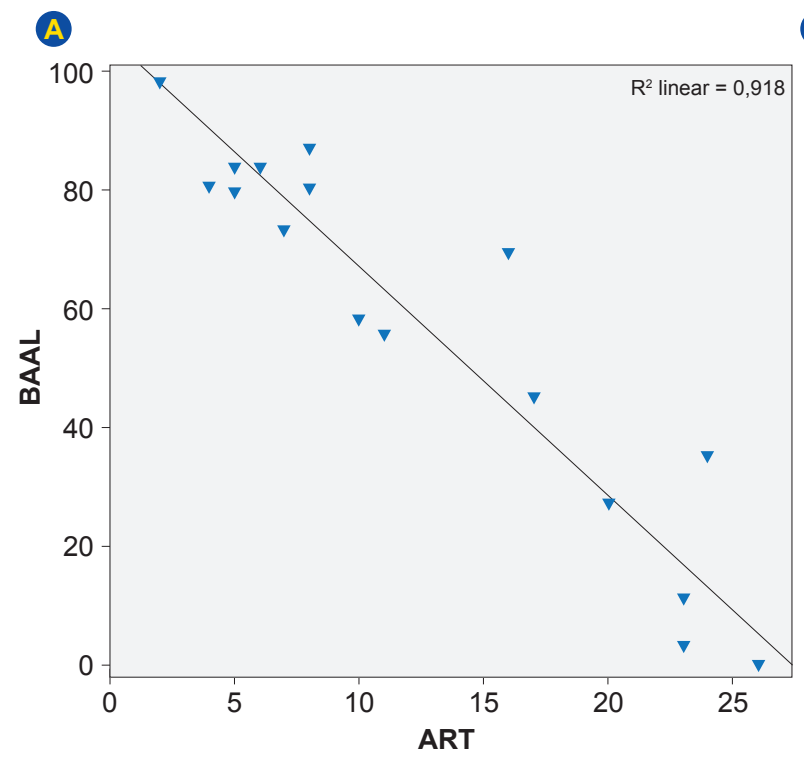

B

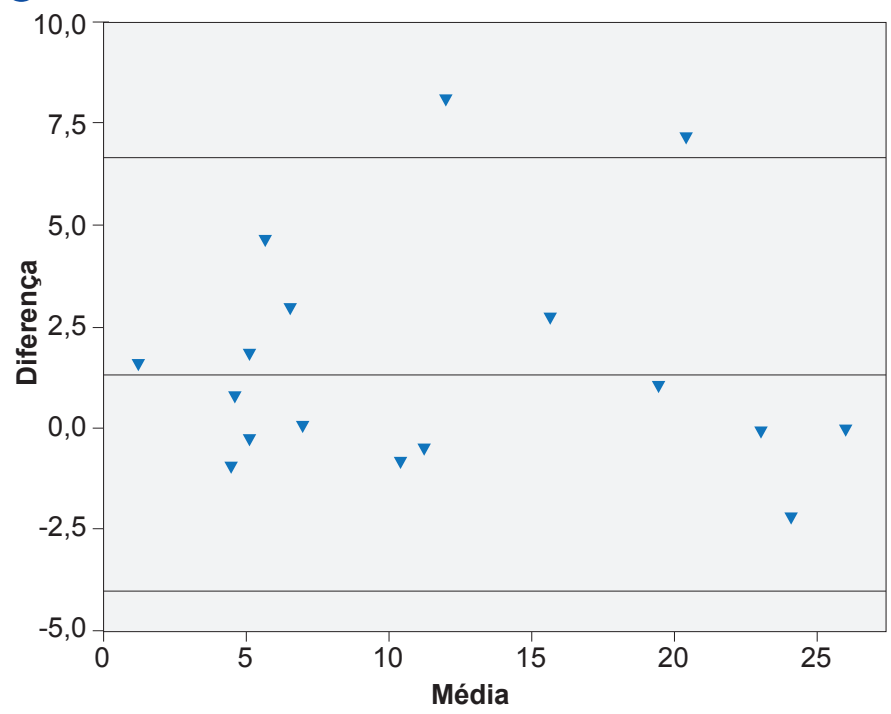

Figura 2 - (A) Distribuição dos valores obtidos para cada doente, comparando valores obtidos com a BAAL e com a ART; (B) Gráfico de Bland-Altman demonstrando a grande concordância entre os valores obtidos na BAAL (ajustados para comparação direta) e na ART.

Para avaliar a capacidade preditiva da escala, foi realizada uma regressão logística binomial (ajustada para idade, escolaridade e NIHSS ${ }_{\text {motor }}$ ). Neste modelo, o ART em D1 foi preditor independente de mau resultado (NIHSS ${ }_{\text {afasia }}$ = 2 - 3) a longo prazo [OR = 1,291, IC 95\% = (1,047, 1,591), $p=0,017$, acuidade $=87,9 \%]$.

\section{DISCUSSÃO}

O ART foi desenhado para quantificar a gravidade da afasia em contexto agudo de AVC avaliando, em menos de três minutos, a compreensão, repetição, nomeação e fluência verbal. A brevidade da sua aplicação é parcialmente explicada pela simplicidade das tarefas, mas também pela rapidez de pontuação nos doentes afásicos agudos, que falham a maioria dos itens. Tal como a NIHSS, é um teste de cabeceira que não requer nenhum material específico e pode ser utilizada por pessoas não especializadas.

Relativamente à adaptação transcultural ao nosso contexto orientámo-nos pelo modelo de avaliação da equivalência da adaptação transcultural de instrumentos sugerido por Herdman et a/ ${ }^{16}$ e que tem vindo a ser empregue em diversos estudos internacionais. Os autores consideram que a equivalência deve ser avaliada a seis níveis: conceptual, de item, semântica, operacional, de mensuração e funcional, tendo operacionalizado as estratégias de avaliação para cada um dos níveis. O processo de adaptação do ART para a população portuguesa respeitou os diferentes níveis apontados, pelo que se pode concluir que a Versão Final Portuguesa do ART apresenta equivalência com a versão original do instrumento.

A simplicidade e rapidez da escala são fundamentais para conseguir a monitorização dos doentes com AVC agudo, que hospitalizados e submetidos a técnicas sucessivas, se cansam facilmente. Na nossa amostra, o tempo médio de aplicação foi de dois minutos e 34,8 segundos, com um máximo de 300 segundos (cinco minutos), tempo semelhante ao referido para a versão original e também comparável com o da aplicação da NIHSS, cuja aplicação média ronda os cinco minutos. ${ }^{5}$

A validação da escala implicou uma análise das características psicométricas essenciais, nomeadamente a consistência interna, validade de construto, acordo interavaliadores e validade concorrente. Em primeiro lugar confirmámos que a escala tem uma boa consistência interna indicada pelo valor elevado do $\alpha$ de Cronbach e pela constatação de que este índice não aumenta com a exclusão

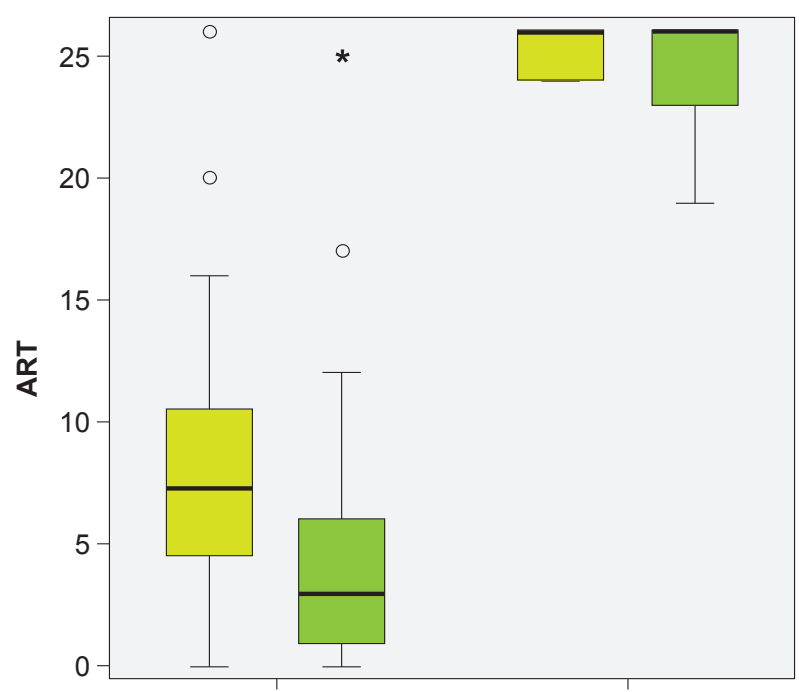

NIHSS 0 ou 1

NIHSS 2 ou 3

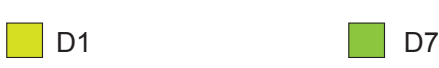

Figura 3 - Divisão dos doentes consoante a pontuação no item 9 no NIHSS (que avalia a linguagem) aos 6 meses. Box plot demonstrando a diferença entre os valores do ART em D1 (verde claro) e D7 (verde escuro) nos grupos com diferente resultado funcional (NIHSS ${ }_{\text {afasia }} \leq 1$ e NIHSS $_{\text {afasia }} \geq 2$ ). 
de nenhum dos subtestes, demonstrando a pertinência dos subtestes utilizados e a sua boa fiabilidade para avaliação geral do domínio da linguagem. Uma vez que os subtestes revelaram correlações moderadas a fortes entre si e com a pontuação final, pudemos igualmente confirmar a sua validade de construto.

A análise do acordo interavaliadores mostrou grande fiabilidade (o coeficiente de concordância entre os dois examinadores foi de 0,985 ), com a variabilidade interobservadores a ser independente da gravidade da afasia (Fig. 2). O grau de acordo interavaliadores revelou-se excelente, com um $\mathrm{k}_{\mathrm{w}}$ de 0,712 , sendo que os $\mathrm{k}_{\mathrm{w}}$ dos subtestes variaram entre 0,811 e 0,956. O gráfico de Bland-Altman (Fig. 1B) mostra que a reprodutibilidade do ART é estável em todos os níveis de gravidade da afasia e que uma diferença de mais de 3,5 pontos indica uma diferença significativa na classificação da gravidade da afasia. Este valor é semeIhante ao da escala de NIHSS. ${ }^{12}$

Estes resultados permitem-nos concluir que a escala pode ser utilizada por diferentes avaliadores ao longo da evolução do doente, facilitando a comunicação e monitorização da gravidade do défice. $A$ análise de correlação dos resultados obtidos no ART e na BAAL, a escala de avaliação de afasias mais utilizada na prática clínica em Portugal, ${ }^{20}$ demonstra boa validade concorrente externa, sugerindo que as provas medem a mesma (dis)função. Tal como no caso das pontuações totais, foi possível observar correlações positivas e estatisticamente significativas entre as pontuações obtidas em todos os subtestes do ART e as funções correspondentes da BAAL. A fluência é o subteste com correlação mais fraca, embora significativa e sem reflexo no resultado final. Tal dever-se-á provavelmente ao facto de na BAAL a fluência ser calculada de maneira diferente para afasias anteriores e posteriores, enquanto no ART, é feita de modo igual para todas. Não tendo obviamente a mesma capacidade e profundidade diagnóstica, este resultado valida o ART enquanto medida diagnóstica de afasia.

Considerando uma variação $\geq 4$ pontos como significativa, $45,2 \%$ dos doentes melhoraram na primeira semana. Este resultado é comparável ao reportado na literatura. ${ }^{21-23}$ A variação do ART na primeira semana é diferente nos grupos de bom e mau prognóstico (Fig. 3). Isto sugere que a escala é adequada à monitorização da afasia, detetando pequenas mudanças iniciais, indicadoras de diferenças a longo prazo com importância funcional. Na prática assistencial atual, o défice de força é avaliado complementarmente pelo NIHSS e pela escala do Medical Research Council, ${ }^{24}$ enquanto a avaliação da afasia se limitava à medida superficial proporcionada pelo NIHSS. Os nossos resultados permitem-nos propor o ART como a escala de avaliação complementar neste domínio da afasia.

A gravidade inicial da afasia é considerada o melhor fator prognóstico da recuperação a longo prazo. ${ }^{25-27}$ Também na nossa amostra, a gravidade inicial avaliada pelo ART se correlacionou com o défice de linguagem na fase crónica, sendo preditor do défice a longo prazo, independentemente da escolaridade, idade e do NIHSS $_{\text {motor }}$ Por cada subida de um ponto no ART, o risco de mau prognóstico é 1,291 vezes maior. Assim, tal como no artigo da escala original, ${ }^{11}$ o ART apresenta valor prognóstico.

O estudo sofre de algumas limitações. Apesar dos resultados obtidos confirmarem a validade desta escala na avaliação da afasia, consideramos que uma amostra mais numerosa poderia dar uma maior robustez estatística aos resultados apresentados. Será também importante no futuro considerar e estudar a aplicação da escala por outro tipo de profissionais envolvidos na assistência de doentes com AVC, nomeadamente os que trabalham em unidades de AVC Agudo (como médicos não neurologistas e enfermeiros especializados).

Relativamente às limitações do instrumento é importante relembrar que o ART é uma escala de gravidade da afasia, criado para ser breve e aplicável em situações urgentes, pelo que a avaliação do defeito de linguagem que proporciona é inevitavelmente superficial, não substituindo uma avaliação pormenorizada através de escalas padronizadas de linguagem. Salienta-se o facto de algumas funções essenciais da linguagem, como a leitura e a escrita, não serem contempladas. O ART não deve ser valorizado isoladamente como uma ferramenta diagnóstica de afasia, uma vez que o desempenho em determinados itens pode ser afetada por distúrbios do discurso não-afásicos (nomeadamente a disartria). Também não deve ser utilizado como instrumento de classificação do tipo de afasia porque, para além da sua brevidade não fazer uma avaliação aprofundada, a avaliação da fluência pode ser prejudicada por disfunção de outros domínios. Contudo, estas limitações são inevitáveis e comuns a outra ferramenta com o mesmo objetivo.

\section{CONCLUSÃO}

O Aphasia Rapid Test é um teste de cabeceira sensível para avaliar a gravidade da afasia. Trata-se de uma ferramenta simples, rápida e com resultados reprodutíveis e que permite uma mensuração e monitorização eficientes da gravidade da afasia na fase aguda e no follow up de doentes com AVC. De salientar que o ART parece ser preditor da evolução da afasia a longo prazo, pelo que poderá contribuir para uma organização mais eficiente e personalizada dos programas de reabilitação do defeito de linguagem de causa vascular.

\section{PROTECÇÃO DE PESSOAS E ANIMAIS}

Os autores declaram que os procedimentos seguidos estavam de acordo com os regulamentos estabelecidos pelos responsáveis da Comissão de Investigação Clínica e Ética e de acordo com a Declaração de Helsínquia da Associação Médica Mundial.

\section{CONFIDENCIALIDADE DOS DADOS}

Os autores declaram ter seguido os protocolos do seu centro de trabalho acerca da publicação de dados. 


\section{CONFLITOS DE INTERESSE}

Os autores declaram não terem qualquer conflito de interesse relativamente ao presente artigo.

\section{REFERÊNCIAS}

1. Pedersen PM, Jorgensen HS, Nakayama H, Raaschou HO, Olsen TS. Aphasia in acute stroke: incidence, determinants, and recovery. Ann Neurol. 1995;38:659-66.

2. Bersano A, Burgio F, Gattinoni M, Candelise L, Group PS. Aphasia burden to hospitalised acute stroke patients: need for an early rehabilitation programme. Int J Stroke. 2009;4:443-7.

3. Musser B, Wilkinson J, Gilbert T, Bokhour BG. Changes in identity after aphasic stroke: implications for primary care. Int J Family Med. 2015;2015:970345.

4. Cameron JI, Cheung AM, Streiner DL, Coyte PC, Stewart DE. Stroke survivor depressive symptoms are associated with family caregiver depression during the first 2 years poststroke. Stroke. 2011;42:302-6.

5. Laska AC, Bartfai A, Hellblom A, Murray V, Kahan T. Clinical and prognostic properties of standardized and functional aphasia assessments. J Rehabil Med. 2007;39:387-92.

6. Enderby P, Crow E. Frenchay Aphasia Screening Test: validity and comparability. Disabil Rehabil. 1996;18:238-40.

7. Flamand-Roze C, Falissard B, Roze E, Maintigneux L, Beziz J, Chacon $A$, et al. Validation of a new language screening tool for patients with acute stroke: the Language Screening Test (LAST). Stroke. 2011;42:1224-9.

8. Nakase-Thompson R, Manning E, Sherer M, Yablon SA, Gontkovsky SL, Vickery C. Brief assessment of severe language impairments: initial validation of the Mississippi aphasia screening test. Brain Inj. 2005;19:685-91.

9. NIHSS - Versão Portuguesa. Porto: Faculdade de Medicina da Universidade do Porto; 2011. [consultado 2014 mar 22]. Disponível em: https://secure.trainingcampus.net/uas/modules/trees/windex. aspx?rx=nihss-portuguese.trainingcampus.net.

10. Adams HP Jr, Davis PH, Leira EC, Chang KC, Bendixen BH, Clarke WR, et al. Baseline NIH Stroke Scale score strongly predicts outcome after stroke: a report of the Trial of Org 10172 in Acute Stroke Treatment (TOAST). Neurology. 1999;53:126-31.

11. Azuar C, Leger A, Arbizu C, Henry-Amar F, Chomel-Guillaume S, Samson Y. The Aphasia Rapid Test: an NIHSS-like aphasia test. J Neurol. 2013; 260:2110-7.

12. Wityk RJ, Pessin MS, Kaplan RF, Caplan LR. Serial assessment of acute stroke using the NIH Stroke Scale. Stroke. 1994;25:362-5.

13. Castro-Caldas A. Diagnóstico e evolução das afasias de causa vascular. Lisboa: Universidade de Lisboa; 1979.

14. Damásio A. Neurologia da linguagem. Lisboa: Universidade de Lisboa;

\section{FONTES DE FINANCIAMENTO}

Os autores declaram não ter recebido subsídios ou bolsas para a elaboração do artigo.

1986.

15. Ferro J. Neurologia do comportamento - estudo da correlação com a tomografia axial computadorizada. Lisboa: Universidade de Lisboa; 1986.

16. Herdman M, Fox-Rushby J, Badia X. A model of equivalence in the cultural adaptation of HRQoL instruments: the universalist approach. Qual Life Res. 1998;7:323-35.

17. New B, Pallier C, Brysbaert M, Ferrand L. Lexique 2: a new French lexical database. Behav Res Methods Instrum Comput. 2004;36:51624.

18. Brysbaert M, New B, Keuleers E. Adding part-of-speech information to the SUBTLEX-US word frequencies. Behav Res Methods. 2012;44:9917.

19. Linguateca. Lisboa: Fundação para a computação Científica Nacional; 2012. [consultado 2014 mar 22]. Disponível em: http://www.linguateca. pt/.

20. Matos M, Jesus L, Cruice M. Assessment of aphasia in Portugal: past, present and future. J Stem-, Spraak- en Taalpathol. 2014;19:S78-83.

21. Inatomi $Y$, Yonehara $T$, Omiya $S$, Hashimoto $Y$, Hirano $T$, Uchino $M$. Aphasia during the acute phase in ischemic stroke. Cerebrovasc Dis. 2008;25:316-23.

22. Maas MB, Lev MH, Ay H, Singhal AB, Greer DM, Smith WS, et al. The prognosis for aphasia in stroke. J Stroke Cerebrovasc Dis. 2012;21:3507.

23. Payabvash S, Kamalian S, Fung S, Wang Y, Passanese J, Kamalian $S$, et al. Predicting language improvement in acute stroke patients presenting with aphasia: a multivariate logistic model using locationweighted atlas-based analysis of admission CT perfusion scans. AJNR Am J Neuroradiol. 2010;31:1661-8.

24. Compston A. Aids to the investigation of peripheral nerve injuries. Medical Research Council: Nerve Injuries Research Committee. Brain. 2010;133:2838-44.

25. Pedersen PM, Vinter K, Olsen TS. Aphasia after stroke: type, severity and prognosis. The Copenhagen aphasia study. Cerebrovasc Dis. 2004;17:35-43.

26. Plowman E, Hentz B, Ellis C Jr. Post-stroke aphasia prognosis: a review of patient-related and stroke-related factors. J Eval Clin Pract. 2012;18:689-94.

27. Wade DT, Hewer RL, David RM, Enderby PM. Aphasia after stroke: natural history and associated deficits. J Neurol Neurosurg Psychiatry. 1986;49:11-6. 